\title{
Single-Dose Memantine Improves Cortical Oscillatory Response Dynamics in Patients with Schizophrenia
}

\author{
Gregory A Light*, ${ }^{*}$, , Wen Zhang', Yash B Joshi', Savita Bhakta', Jo A Talledo' and Neal R Swerdlow' \\ 'VISN-22 Mental Illness Research Education and Clinical Center, VA San Diego Healthcare System, San Diego, CA, USA; ${ }^{2}$ Department of \\ Psychiatry, University of California San Diego, La Jolla, CA, USA
}

\begin{abstract}
Aberrant gamma-band $(30-80 \mathrm{~Hz})$ oscillations may underlie cognitive deficits in schizophrenia (SZ). Gamma oscillations and their regulation by NMDA receptors can be studied via their evoked power $(\gamma E P)$ and phase locking $(\gamma \mathrm{PL})$ in response to auditory steady-state stimulation; these auditory steady-state responses (ASSRs) may be biomarkers for target engagement and early therapeutic effects. We previously reported that memantine, an NMDA receptor antagonist, enhanced two biomarkers of early auditory information processing: prepulse inhibition and mismatch negativity (MMN) in SZ patients and healthy subjects (HS). Here, we describe memantine effects on $\gamma E P$ and $\gamma \mathrm{PL}$ in those subjects. SZ patients $(n=18)$ and HS $(n=14)$ received memantine $20 \mathrm{mg}$ (p.o.) and placebo over 2 test days in a doubleblind, randomized, counterbalanced, cross-over design. The ASSR paradigm (I ms, 85 dB clicks in 250-0.5 s trains at a frequency of $40 \mathrm{~Hz}$; $0.5 \mathrm{~s}$ inter-train interval) was used to assess $\gamma \mathrm{EP}$ and $\gamma \mathrm{PL}$. SZ patients had reduced $\gamma \mathrm{EP}$ and $\gamma \mathrm{PL}$; memantine enhanced $\gamma \mathrm{EP}$ and $\gamma \mathrm{PL}$ $(p<0.025$ and 0.002 , respectively) in both $\mathrm{SZ}$ and HS. In patients, significant correlations between age and memantine effects were detected for $\gamma \mathrm{EP}$ and $\gamma \mathrm{PL}$ : greater memantine sensitivity on $\gamma \mathrm{EP}$ and $\gamma \mathrm{PL}$ were present in younger SZ patients, similar to our reported findings with MMN. Memantine acutely normalized cortical oscillatory dynamics associated with NMDA receptor dysfunction in SZ patients. Ongoing studies will clarify whether these acute changes predict beneficial clinical, neurocognitive and functional outcomes. These data support the use of gamma-band ASSR as a translational end point in pro-cognitive drug discovery and early-phase clinical trials. Neuropsychopharmacology (2017) 42, 2633-2639; doi:10.1038/npp.2017.8I; published online 31 May 2017
\end{abstract}

\section{INTRODUCTION}

Patients with schizophrenia (SZ) have deficits in many domains, ranging from early sensory processing to executive function, which contribute to impairments in cognitive and psychosocial functioning (Thomas et al, 2017). Neural oscillations in the $30-50 \mathrm{~Hz}$ range (centered near $40 \mathrm{~Hz}$ and referred to as the 'gamma band') appear to reflect a fundamental CNS resonance frequency that is critical for communication across multiple brain regions (Traub et al, 1996). Gamma-band oscillations are transiently generated during a wide range of cognitive operations such as sensory discrimination, learning, and memory-all domains in which many SZ patients have deficits (for reviews, see Ford and Mathalon, 2008; McNally and McCarley, 2016; Salisbury et al, 2017; Spencer et al, 2004).

In recent years, the response to auditory steady-state stimulation measured in the electroencephalogram (EEG) has emerged as a leading candidate biomarker of disease process, therapeutic effect, and the capacity of neural circuits to support gamma-band oscillations under 'optimized'

*Correspondence: Dr GA Light, Department of Psychiatry, University of California San Diego, UC San Diego School of Medicine, San Diego, CA 92093-0804, USA, Tel: 619543 2496, Fax: 619543 2493, E-mail: glight@ucsd.edu

Received 26 January 2017; revised 10 April 2017; accepted II April 2017; accepted article preview online 20 April 2017 (ie, stimulus-driven) conditions (O'Donnell et al, 2013; Sun et al, 2011; Light et al, 2006; Kirihara et al, 2012). In this auditory steady-state response (ASSR) paradigm, clicks or amplitude-modulated tones are presented at a rate of $40 \mathrm{~Hz}$; the resulting stimulus-driven gamma power and phase synchronization in the EEG is thought to reflect the capacity of the auditory system to generate oscillations in synchrony with the stimuli. As described in Thuné et al (2016), 20 studies have assessed ASSR dynamics in SZ patients using a variety of stimulation and analytic procedures; 17 of these studies identified impairment in both evoked power and phase synchronization. Notably, many of these studies also identified significant correlations between ASSR oscillatory dynamics and clinical symptoms (eg, auditory hallucinations: Hirano et al, 2015) and cognitive test performances (eg, working memory: Light et al, 2006; verbal learning: Kirihara et al, 2012).

While the neural mechanisms of ASSR remain unclear, the involvement of parvalbumin-positive GABAergic basket interneurons and the pyramidal cells of the upper layers of sensory cortex is well documented (review Featherstone et al, 2015; Sivarao et al, 2016). Within the circuitry, $N$-methyl-Daspartic acid (NMDA) receptor function appears to be critical for EEG synchronization in the gamma frequency range. NMDA hypofunction is implicated in the pathophysiology of SZ (Javitt, 2012), and NMDA antagonists have been shown to modulate ASSR in a dose-dependent manner 
in preclinical studies (Hiyoshi et al, 2014). NMDA antagonists also augment the $40 \mathrm{~Hz}$ ASSR response in rodents expressing a SZ-linked phenotype (Phillips et al, 2012). While animal models of SZ suggest the feasibility of pharmacologically regulating ASSR in patients with SZ via drugs acting at NMDA receptors, few studies have addressed the effect of NMDA alterations on ASSR in human subjects, none involving patients with SZ.

Memantine (MEM) is an uncompetitive NMDA receptor antagonist, with low affinity but rapid blocking and unblocking ability; its minimal impact on basal NMDA transmission distinguishes it mechanistically from other NMDA antagonists (Lipton, 2006). Its main clinical indication is Alzheimer's disease, however, it has positive activity in 'cognitive' measures in both healthy animals and a range of animal models, including those for dementia, depression, ischemia, and neuroinflammation (cf. Ma et al, 2015). While its mechanism of therapeutic action remains unclear, MEM enhances hippocampal long-term potentiation (LTP), and reverses the loss of LTP under various experimental conditions; it also alters excitation/inhibition (E/I) dynamics in frontal circuitry implicated in models of SZ neuropathology (Povysheva and Johnson, 2016).

We previously demonstrated that MEM enhanced sensorimotor gating - as measured by prepulse inhibition (PPI) of startle-and mismatch negativity (MMN) in patients with SZ and healthy comparison subjects (HS) (Swerdlow et al, 2016). These two measures index pre-attentive information processing and are each positively associated with both neurocognition and daily life functioning (Giakoumaki et al, 2006; Light et al, 2007; Swerdlow et al, 2006; Thomas et al, 2017; Rissling et al, 2014). Here we report findings of ASSR measures in these subjects, after acute administration of MEM (20 mg, p.o.) or placebo in a double-blind, withinsubject cross-over design.

\section{MATERIALS AND METHODS}

All procedures were approved by the UCSD Human Subject Institutional Review Board. Participants were 18-50-year-old adult $\mathrm{HS}$ and patients with a clinically stable chronic psychotic disorder (SZ, $n=17$; schizoaffective disorder, depressed, $n=1$ ) who met the inclusion criteria for this study (Table 1).

Subjects who passed a phone checklist were invited for an in-person screening session that included an urine toxicology test, hearing test (Saico Audiometer, Assens Denmark), a startle reflex test to confirm adequate reflex magnitude, and questionnaires related to demographic and clinical information (Table 2). Diagnoses in CPD patients were confirmed by the MINI International Neuropsychiatric Interview (6.0) (Sheehan et al, 1998), and symptom severity was assessed with the positive and negative syndrome scale (Kay et al, 1987). Participants who met the study inclusion criteria returned for testing after 6-10 days, and for a second test 7 days later. Testing followed a double-blind, placebocontrolled, pseudo-randomized balanced drug order design. CPD subjects maintained their medication doses during the study. Subjects arrived at $\sim 0830$ on the test day, ate a standardized breakfast, and completed urine toxicology and hearing tests. At $0900, \mathrm{MEM} \mathrm{HCl}(20 \mathrm{mg})$ or placebo was
Table I Demographic and Clinical Characteristics of Participants

\begin{tabular}{lcc}
\hline & $\begin{array}{c}\text { Healthy } \\
\text { subjects }\end{array}$ & Schizophrenia patients \\
\hline Age (mean, range) & $26.64(19-36)$ & $37.22(21-48)^{\mathrm{a}}$ \\
M: F & $10: 4$ & $11: 7$ \\
Smoke : non-smoker & $0: 14$ & $7: 11^{\mathrm{b}}$ \\
WRAT reading (mean, SEM) & $103.89(3.99)$ & $93.78(2.52)^{\mathrm{b}}$ \\
GAF (mean, SEM) & - & $56.89(1.10)$ \\
Illness duration (mean, range) & - & $21.18(6-34)$ \\
Chlorpromazine equiv (mean, & - & $586.73(122.43)$ \\
SEM) & - & $8.28(1.78)$ \\
Anticholinergic equiv (mean, & & \\
SEM) & & $17.56(1.10)$ \\
PANSS & & $17.39(1.85)$ \\
Positive & & $31.00(2.45)$ \\
$\quad$ Negative & & \\
General &
\end{tabular}

${ }^{\mathrm{a}}$ Significant group differences ( $p<0.01$, two-tailed).

bSignificant group differences ( $p<0.05$, two-tailed)

administered (p.o.) in either healthy controls or SZ patients. An initial group of patients and HS were tested with a lower MEM dose $(20 \mathrm{mg})$; however, as previously reported (Swerdlow et al, 2016), this lower dose produced no measurable changes in subjective ratings, autonomic measures, PPI, or MMN, and this inactivity was confirmed for ASSR measures (no significant MEM main or interaction effects in the $10 \mathrm{mg}$ group were detected with any ASSR parameters). Since the $10 \mathrm{mg}$ dose appears to be inert in all of our laboratory measures, only data from the $20 \mathrm{mg}$ dose group are presented here. At 1130, subjects received a standardized lunch.

Neurophysiological measures were timed to coincide with peak blood levels of MEM after a single oral dose of $20 \mathrm{mg}$ (Sonkusare et al, 2005). Startle testing began $210 \mathrm{~min}$ after pill administration, and $345 \mathrm{~min}$ post-pill, MMN testing commenced; findings from both PPI and MMN measures were reported previously (Swerdlow et al, 2016). ASSR measures followed immediately after MMN testing, beginning about 385 min post-pill.

The ASSR paradigm utilized $1 \mathrm{~ms}, 85 \mathrm{~dB}$ clicks presented in $500 \mathrm{~ms}$ trains at a frequency of $40 \mathrm{~Hz}$. A total number of 250 click trains were played with an inter-train interval of $0.5 \mathrm{~s}$. The auditory stimuli were delivered through insert earphones. Participants were instructed to ignore auditory stimuli while staring at a fixation cross at the center of the screen. The ASSR recording lasted approximately for $4 \mathrm{~min}$ (Supplementary Figure 1).

Electroencephalographic (EEG) data were continuously recorded with a 64-channel BioSemi ActiveTwo system at a sampling rate of $2048 \mathrm{~Hz}$. Data processing was performed offline using Matlab, EEGlab, and BrainVision Analyzer and as per established methods (Kirihara et al, 2012; Light et al, 2006). Briefly, an averaged reference was applied to the EEG recordings; vertical and horizontal eye movement artifacts were corrected using the independent component analysis. Continuous data were then segmented relative to the onset of 
Table 2 Significant Relationships were Detected between MEM Effects on Gamma Evoked Power and Phase Locking and Age in Schizophrenia Patients (Supplementary Figures 2); by Including Data Previously Reported from Measures of Mismatch Negativity and Prepulse Inhibition (Swerdlow et al, 2016), Significant Relationships were also Evident between MEM Effects on $\gamma$ EP and MMN and Between $\gamma$ PL and PPI

\begin{tabular}{lccc}
\hline & Age & MEM effect on $\gamma$ EP & MEM effect on $\gamma$ PL \\
\hline MEM effect on $\gamma$ EP & $-0.71^{\mathrm{a}}$ & & \\
MEM effect on $\gamma \mathrm{PL}$ & $-0.63^{\mathrm{a}}$ & $0.74^{\mathrm{a}}$ & 0.26 \\
MEM effect on MMN & $-0.56^{\mathrm{b}}$ & $0.54^{\mathrm{b}}$ & $-0.59^{\mathrm{b}}$ \\
MEM effect on PPI & $0.54^{\mathrm{b}}$ & -0.51 & $-0.70^{\mathrm{a}}$ \\
\hline
\end{tabular}

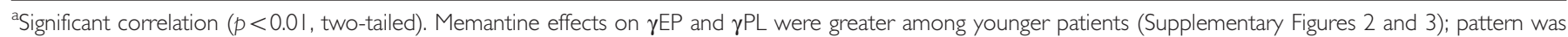
previously reported for MMN, while the opposite pattern (greater memantine effects among older patients) was reported for PPI (Swerdlow et al, 20I6).

Significant correlation ( $p<0.05$, two-tailed).
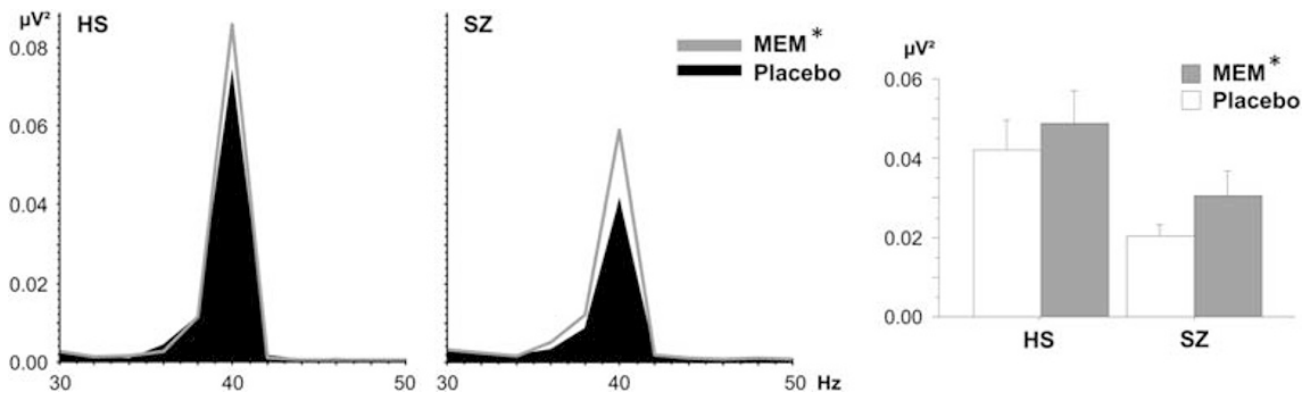

Figure I Gamma evoked power (EP) in healthy subjects (left) and schizophrenia patients (right) in placebo (black) and 20 mg MEM (red) conditions. In the placebo condition, schizophrenia patients show significant reductions in EP relative to healthy subjects, consistent with previous reports. *MEM significantly increases EP in both groups; in schizophrenia patients, MEM increased EP to levels that did not differ statistically from healthy subjects after placebo ( $F=1.54$, df I.30, NS). A full color version of this figure is available at the Neuropsychopharmacology journal online.

the stimuli ( $-100 \mathrm{~ms}$ to $500 \mathrm{~ms}$ ), and each epoch was baseline-corrected relative to the $100 \mathrm{~ms}$ pre-stimulus interval. Epochs containing $\pm 70 \mu \mathrm{V}$ were automatically rejected.

Gamma evoked power $(\gamma \mathrm{EP})$ and phase locking $(\gamma \mathrm{PL})$ were assessed based on the first 100 artifact-free epochs at Fz. For $\gamma \mathrm{EP}$ analyses, the averaged epochs across the click trains were transformed into power spectrum by means of fast Fourier transform (FFT) using a bin width of $2 \mathrm{~Hz}$. The $40 \mathrm{~Hz}$ power spectrum was averaged across $4 \mathrm{~Hz}$ band from 38 to $42 \mathrm{~Hz}$.

$\gamma \mathrm{PL}$ was calculated on wavelet coefficients obtained from Morlet wavelet transformation of the segmented data (frequencies represented from 5 to $95 \mathrm{~Hz}$, with a total number of 45 frequency layers); $\gamma \mathrm{PL}$ was estimated by extracting and averaging across the $30-50 \mathrm{~Hz}$ frequency layer. $\gamma \mathrm{PL}$ quantifies consistency of oscillatory phase across individual trials, ranging from 0 (purely non-phase-locked activity) to 1 (fully phase-locked activity). For statistical analyses, mean values were obtained for each of the six $100 \mathrm{~ms}$ windows from -100 to $500 \mathrm{~ms}$ relative to stimulus onset.

Symptom rating scales (SRS) were assessed before, and 30, 90, 150, 200, and $230 \mathrm{~min}$ after pill consumption, as previously described (eg, Swerdlow et al, 2009; see Supplementary Methods for details).

Data were analyzed by RM-ANOVA, with diagnosis as a between-subject factor, and drug condition (placebo $v s$ active) as a within-subject factor for measures of $\gamma \mathrm{EP}$ and $\gamma$ PL. Initial analyses revealed robust and time binindependent effects of diagnosis and drug across the 200-500 ms stimulation window, and thus this interval was the focus of all subsequent analyses, including correlations with clinical and experimental variables. SRS and autonomic measures were continuous variables and analyzed by ANOVA as 'change scores' (difference from pre-pill baseline). These change score calculations of MEM effects on MMN were scaled so that positive values indicate greater MEM effect, consistent with $\gamma \mathrm{EP}, \gamma \mathrm{PL}$, and PPI.

Study recruitment did not balance groups for sex, age, or medication status, but where possible, these variables were examined post hoc to determine their potential impact on MEM effects. Age-matched subgroups of patients and HS were formed using the 10 youngest patients and the 10 oldest HS. Drug order (placebo test 1 vs active test 1) was also assessed as a potential moderator of drug effects. For all statistical comparisons, alpha was 0.05 for two-tailed contrasts and 0.10 for one-tailed contrasts.

\section{RESULTS}

Demographic and clinical characteristics of the final cohort are shown in Table 1 . Most (16/18) patients were taking atypical antipsychotics (APs), one was taking only typical APs and one was unmedicated at the time of testing. On average, patients were significantly older than HS $(p<0.0001)$.

Analyses of autonomic measures revealed no significant main or interaction effects of diagnosis or MEM dose on change in heart rate, diastolic or systolic blood pressure. SRS data generally yielded no meaningful main or interaction effects. Thus, consistent with our past experience, MEM 

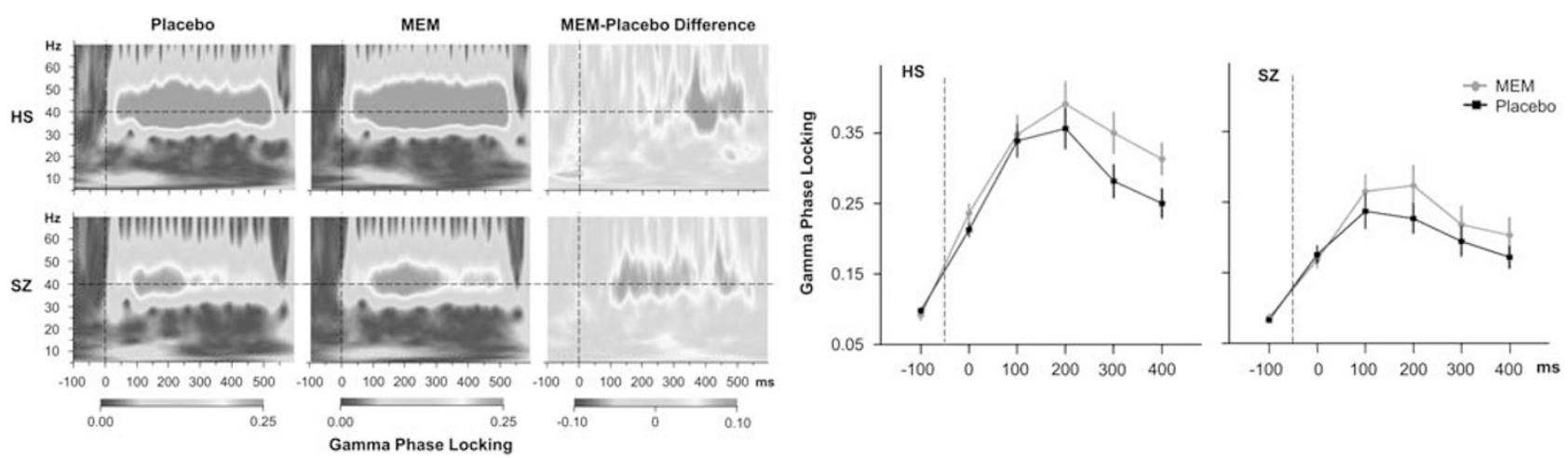

Figure 2 Gamma phase locking (PL): time-frequency plots in healthy subjects (top row) and schizophrenia patients (bottom row) during placebo (left panel), $20 \mathrm{mg}$ MEM (middle panel), and MEM-placebo difference (right panel). The $x$ axis indicates time in ms and the $y$ axis indicates frequency. Color indicates phase locking factors. The right panel shows mean gamma PL in healthy subjects (left) and schizophrenia patients (right) during placebo (black) and $20 \mathrm{mg}$ MEM (red) across 100 ms time bins from - 100 to $500 \mathrm{~ms}$. In the placebo condition, schizophrenia patients show significant reductions in PL relative to healthy subjects, consistent with previous reports. MEM significantly increases PL in both groups; in schizophrenia patients, MEM increased PL to levels that did not differ statistically from healthy subjects after placebo $(F=3.21$, df I.30, NS). A full color version of this figure is available at the Neuropsychopharmacology journal online.

(20 mg p.o.) had minimal effects on autonomic and subjective measures (Swerdlow et al, 2016; Bhakta et al, 2016).

RM-ANOVA revealed that patients with SZ had significantly reduced $\gamma \mathrm{EP}$ (Figure 1) and $\gamma \mathrm{PL}$ (Figure 2), consistent with previous reports (main effect of diagnosis- $\gamma \mathrm{EP}$ : $F=5.89$, df $1.30, \quad p<0.025 ; \quad \gamma \mathrm{PL}: \quad F=11.83$, df 1.30, $p<0.002)$. This effect on phase locking was independent of time bins (diagnosis $\times$ bin interaction: $F=1.62$, df 2.60 , NS; Figure 2), and was evident with subgroups of age-matched HS and patients $(p<0.025)$. MEM significantly enhanced $\gamma \mathrm{EP}$ and $\gamma \mathrm{PL}$ in both SZ patients and HS (main effects of drug- $\gamma \mathrm{EP}: F=6.40$, df $1.30, p<0.02 ; \gamma \mathrm{PL}: F=10.42$, df 1.30 , $p<0.004)$. There was no significant interaction of diagnosis $\times$ drug for either $\gamma \mathrm{EP}$ or $\gamma \mathrm{PL}$ (both $F \mathrm{~s}<1$ ), suggesting that MEM effects on these measures were not specific for diagnosis.

MEM effects on both $\gamma \mathrm{EP}$ and $\gamma \mathrm{PL}$ were independent of test order (both drug $\times$ test order interactions NS) and were also significant when groups were matched for age as above (main effects of drug: $p$ 's $<0.007$ and 0.003 , respectively). While group differences in MEM-associated enhancement of $\gamma \mathrm{EP}$ and $\gamma \mathrm{PL}$ were evident when groups were age-matched, the magnitudes of MEM effects on both of these measures (difference score: MEM minus placebo) were significantly and negatively correlated with age in SZ patients $(r$ 's $=-0.71$ and -0.63 ; $p$ 's $<0.0015$ and 0.005 , respectively). Greater MEM sensitivity in younger patients reproduces the pattern previously detected for $\mathrm{MMN}$, while the opposite pattern (greater MEM sensitivity in older patients) was previously reported for PPI (Table 2, Supplementary Figures 2); (Swerdlow et al, 2016). This MEM effect difference score in patients did not correlate significantly with other clinical measures, including age of illness onset, symptom severity, chlorpromazine equivalents, or estimated level of anticholinergic activity.

\section{DISCUSSION}

In this study, a single $20 \mathrm{mg}$ dose of MEM significantly increased gamma evoked power and phase synchronization in patients with SZ and HS. Preclinical studies have shown that MEM enhances spontaneous gamma power in rodents via a linear, positive dose-response effect; by contrast, other NMDA antagonists-PCP, ketamine, and MK801-exhibit inverted-U dose-response functions with respect to spontaneous gamma power in rodents, and also adversely impact other frequency bands (Hiyoshi et al, 2014).

Several studies have linked higher levels of early auditory information processing (EAIP), including gamma power and phase locking, with better clinical and functional outcomes in SZ patients (Light et al, 2006; Kirihara et al, 2012; Thomas et al, 2017). On the basis of these reports, the simplest interpretation of the present findings-and those of MEMenhanced PPI and MMN previously published by our group (Swerdlow et al, 2016)—might suggest that MEM should have clinical and functional benefits in SZ patients. Studies of MEM effects on neurocognition and symptoms in SZ, however, have yielded conflicting results.

One large study (Lieberman et al, 2009) reported 'negative' effects of MEM in SZ patients: an 8-week double-blind, randomized clinical trial (RCT) of MEM $(20 \mathrm{mg} / \mathrm{d} ; n=70) v s$ placebo $(n=68)$ added to atypical APs found no change in positive or negative symptoms or brief assessment of cognition in SZ scores. Unfortunately, this early 'negative' study has shaped much of the field's view of MEM as a nonefficacious adjunct to antipsychotics in SZ. At least six placebo-controlled studies and several large open-label reports, however, have demonstrated benefits of MEM in SZ. A 12-week double-blind RCT of MEM vs placebo added to existing antipsychotics $(n=64)$ (Omranifard et al, 2015) found enhanced function and life quality in both groups, with significantly greater gains in the MEM group ( $p$ 's <0.001). Another placebo-controlled double-blind 12-week cross-over study of MEM effects in clozapinerefractory SZ patients $(n=52)$ found significant gains in cognition and symptoms, with further symptom improvement by 26 and 52 weeks (Veerman et al, 2016a,b). A PBO- 
controlled double-blind 12-week trial of MEM $(n=18)$ vs placebo $(n=18)$ added to antipsychotics found significant benefits of MEM over placebo for negative symptoms $(p=0.003)$ and Mini-Mental State Exam (MMSE; $p<0.001)$ (Mazinani et al, 2017). An 8-week double-blind placebocontrolled study of MEM added to risperidone in $40 \mathrm{SZ}$ patients reported significant MEM-associated improvement in negative symptoms $(p<0.001)$ (Rezaei et al, 2013). Another double-blind study found large reductions in positive and negative symptoms $(d=1.38-3.33)$ and improved MMSE after 12 weeks of MEM $(20 \mathrm{mg} / \mathrm{d} ; n=10) v s$ placebo $(n=11)$ added to clozapine (de Lucena et al, 2009). A smaller, 6-week, open-label study of MEM (5-20 mg/d) in SZ inpatients reported significant improvement in positive and negative symptoms but not cognition (Krivoy et al, 2008). A chart-based retrospective case series found improved positive and/or negative symptoms as well as cognitive and/or functional domains in 17 out of $26 \mathrm{SZ}$ patients when MEM was added to an antipsychotic (John et $a l, 2014)$; in several patients, antipsychotic dose requirements also declined with MEM. Many case reports describe symptom reduction and function gains after MEM (5-20 mg/d) in SZ patients; in some, symptoms returned when MEM was stopped and resolved after MEM was restarted (Zdanys and Tampi, 2008; Matsuda et al, 2013; Paraschakis, 2014).

Importantly, none of the above studies utilized this putative pro-cognitive agent in concert with any systematic cognitive intervention, nor were any specific biomarkers employed to predict sensitive subgroups of patients. Both of these factors-the use of a contemporaneous cognitive intervention to engage the MEM-induced information processing gains, and the use of predictive biomarkers to identify patients with a 'MEM-sensitive' phenotype-might be important considerations in future therapeutic models with MEM and other putative pro-cognitive therapeutics in $\mathrm{SZ}$, as described in previous reports from our group (Swerdlow, 2011; Light and Swerdlow, 2014; Swerdlow et al, 2016; Swerdlow et al, in press; Perez et al, in press).

In considering the relationship between acute MEMenhanced EAIP and the potential benefits of MEM in SZ therapeutics, two other issues are quite relevant. First, acute MEM does not enhance neurocognition in SZ patients (Bhakta et al, 2016). Thus, there is a dissociation between the immediate EAIP-enhancing effects of MEM and its impact on neurocognition. Certainly, this dissociation could reflect deleterious acute effects of a single $20 \mathrm{mg}$ dose of MEM on neurocognition (NB: not detected in Bhakta et al, 2016), which might wane with repeated administration, especially via a gradual titration from lower to higher doses. Alternatively, the mechanisms of MEM-enhanced EAIP may be such that gains in neurocognition would be predicted as a later but not immediate consequence, much like the impact of a hearing aid on speech development in a preverbal hearing-impaired child. In other words, we hypothesize that the lack of immediate MEM-enhanced gains in neurocognition reflects the acute nature of this measurement (4-6h post-MEM) vs the time and experience required in order for enhanced EAIP and subsequent improvements in higher-order auditory processing (eg, speed and fidelity) to produce changes in neurocognition and its underlying forebrain substrates. A second issue, however, is that we cannot assume that EAIP-enhancing effects of MEMevident after a single dose-would be sustained with longterm daily MEM dosing. To address this issue, we are currently pursuing measures of EAIP during sustained MEM administration in SZ patients.

Another important but unanswered question is whether acute EAIP-enhancing effects of MEM can serve as a biomarker predicting later clinical benefits of MEM. Clearly, if MEM acutely increases gamma power and phase locking in a specific SZ patient, this suggests that MEM is accessing neural mechanisms associated with cortical oscillatory dynamics in a way that 'normalizes' these processes. Such evidence of 'neuroactivity' suggests that this individual might be more sensitive to the potential salutary effects of MEM, compared, for example, to an individual in whom MEM produces no brain 'signal'.

The present findings also underscore potential heterogeneity in the MEM 'brain signal' in SZ patients, which may be quite relevant to its use as a predictive biomarker. While MEM 'normalizes' four different EAIP measures, these effects are not uniform within any individual patient: strong positive correlations are evident among MEM-induced changes in MMN and gamma power, and strong negative correlations are evident between PPI and MMN (Swerdlow et al, 2016) and between PPI and gamma phase locking (present study). Furthermore, MEM-enhancement of MMN, gamma power, and phase locking are negatively associated with patient age, while MEM-enhanced PPI is positively associated with patient age. It is thus conceivable that MEM might have differential effects on brain function in SZ patients as a function of age, illness duration, or baseline deficits in different forms of EAIP. Our group also reported preliminary evidence for an association between MEM effects on both PPI and neurocognition, and a singlenucleotide polymorphism in the gene encoding the glutamate ionotropic receptor subtype 3 A (rs1337696; Bhakta et $a l, 2016)$. Others have reported an association between MEM sensitivity in humans and rodents, and genetic variations in glutamate ionotropic receptor subtypes $2 \mathrm{~A}$ and 2D (GRIN2A, GRIN2D) (Lesca et al, 2013; Li et al, 2016; Pierson et al, 2014). Thus, genes regulating glutamate receptor function may also help predict a therapeutic response to MEM in SZ patients.

Results of this study should be considered in the context of several limitations. First, psychotropic medications were not experimentally controlled. As in many studies of SZ, patients were prescribed heterogeneous and complex medication regimens that included medications that could impact biomarker sensitivity to acute MEM effects. Second, sample size was modest. The small number of subjects prevents us from disentangling medications, genes, and demographic factors (eg, age) that may contribute to individual variability in ASSR parameters. Third, only a single active dose of MEM was reported here. In fact, we previously reported that a lower MEM dose (10 mg, p.o.) produced no measurable changes in any measures, including subjective ratings, autonomic measures, PPI, or MMN (Swerdlow et al, 2016). Indeed, initial tests of the present gamma-band measures with $10 \mathrm{mg}$ MEM also detected no evidence of bioactivity, and including those subjects in the present analyses yielded results leading to the identical conclusions reported here: that the higher dose of MEM (20 mg) significantly enhanced 
gamma-band measures in patients and HS. Fourth, patients were, on average, significantly older than healthy subjects. Despite this fact, patient deficits in gamma-band responses were still detected in subgroups carefully matched for age; with the broader age range among patients, it was possible to detect important age-related MEM effects on gamma-band responses.

Finally, it is worth considering the neural mechanisms that underlie acute MEM-induced increases in gamma power and phase locking. Thus, enhanced gamma power and phase locking might reflect gains in the speed and/or fidelity of auditory processing based on MEM effects at levels ranging from the brain stem (Tarasenko et al, 2014) up to thalamocortical projections, and many interceding loci. We are beginning to probe the relationship of signal fidelity at these different levels of auditory processing and neurocognitive gains in SZ (Rackelmann et al, 2016; Perez et al, 2017; Thomas et al, 2017). Identifying the mechanisms, or at least the neural locus (loci), of MEM-enhanced EAIP in SZ might have important implications both for our understanding of the role of NMDA function in the pathophysiology of SZ and for the development of future therapeutics (Light and Swerdlow, 2014, 2015a,b).

\section{FUNDING AND DISCLOSURE}

GAL has served as a consultant for Astellas, Boehringer Ingelheim, Heptares, Merck, NeuroSig, Neuroverse, and Takeda. The remaining authors declare no conflict of interest.

\section{ACKNOWLEDGMENTS}

This work was supported by the National Institute of Mental Health (MH-059803, MH094320, and MH101072), the Brain and Behavior Research Foundation, the VISN-22 Mental Illness Research, Education, and Clinical Center (MIRECC), and the Sidney R Baer, Jr Research Foundation.

\section{REFERENCES}

Bhakta SG, Chou HH, Rana B, Talledo JA, Balvaneda B, Gaddis L et al (2016). Effects of acute memantine administration on MATRICS consensus cognitive battery performance in psychosis: testing an experimental medicine strategy. Psychopharmacology 233: 2399-2410.

de Lucena D, Fernandes BS, Berk M, Dodd S, Medeiros DW, Pedrini $M$ et al (2009). Improvement of negative and positive symptoms in treatment-refractory schizophrenia: a double-blind, randomized, placebo-controlled trial with memantine as add-on therapy to clozapine. J Clin Psychiatry 70: 1416-1423.

Featherstone RE, McMullen MF, Ward KR, Bang J, Xiao J, Siegel SJ (2015). EEG biomarkers of target engagement, therapeutic effect, and disease process. Ann N Y Acad Sci 1344: 12-26.

Ford JM, Mathalon DH (2008). Neural synchrony in schizophrenia. Schizophr Bull 34: 904-906.

Giakoumaki SG, Bitsios P, Frangou S (2006). The level of prepulse inhibition in healthy individuals may index cortical modulation of early information processing. Brain Res 1078: 168-170.

Hirano Y, Oribe N, Kanba S, Onitsuka T, Nestor PG, Spencer KM (2015). Spontaneous gamma activity in schizophrenia. JAMA Psychiatry 72: 813-821.
Hiyoshi T, Kambe D, Karasawa J-I, Chaki S (2014). Differential effects of NMDA receptor antagonists at lower and higher doses on basal gamma band oscillation power in rat cortical electroencephalograms. Neuropharmacology 85: 384-396.

Javitt DC (2012). Twenty-five years of glutamate in schizophrenia: are we there yet? Schizophr Bull 38: 911-913.

John JP, Lukose A, Manjunath S (2014). Off-label use of memantine as adjunctive treatment in schizophrenia: a retrospective case series study. Pharmacopsychiatry 47: 202-209.

Kay SR, Fiszbein A, Opler LA (1987). The positive and negative syndrome scale (PANSS) for schizophrenia. Schizophr Bull 13: 261-276.

Kirihara K, Rissling AJ, Swerdlow NR, Braff DL, Light GA (2012). Hierarchical organization of gamma and theta oscillatory dynamics in schizophrenia. Biol Psychiatry 71: 873-880.

Krivoy A, Weizman A, Laor L, Hellinger N, Zemishlany Z, Fischel T (2008). Addition of memantine to antipsychotic treatment in schizophrenia inpatients with residual symptoms: a preliminary study. Eur Neuropsychopharmacol 18: 117-121.

Lesca G, Rudolf G, Bruneau N, Lozovaya N, Labalme A, Boutry-Kryza N et al (2013). GRIN2A mutations in acquired epileptic aphasia and related childhood focal epilepsies and encephalopathies with speech and language dysfunction. Nat Genet 45: 1061-1066.

Li D, Yuan H, Ortiz-Gonzalez XR, Marsh ED, Tian L, McCormick EM et al (2016). GRIN2D recurrent de novo dominant mutation causes a severe epileptic encephalopathy treatable with NMDA receptor channel blockers. Am J Hum Genet 99: 802-816.

Lieberman JA, Papadakis K, Csernansky J, Litman R, Volavka J, Jia XD et al (2009). A randomized, placebo-controlled study of memantine as adjunctive treatment in patients with schizophrenia. Neuropsychopharmacology 34: 1322-1329.

Light GA, Hsu JL, Hsieh MH, Meyer-Gomes K, Sprock J, Swerdlow NR et al (2006). Gamma band oscillations reveal neural network cortical coherence dysfunction in schizophrenia patients. Biol Psychiatry 60: 1231-1240.

Light GA, Swerdlow NR (2014). Neurophysiological biomarkers informing the clinical neuroscience of schizophrenia: mismatch negativity and prepulse inhibition of startle. Curr Top Behav Neurosci 21: 293-314.

Light GA, Swerdlow NR (2015a). Future clinical uses of neurophysiological biomarkers to predict and monitor treatment response for schizophrenia. Ann N Y Acad Sci 1344: 105-119.

Light GA, Swerdlow NR (2015b). Bending the curve on psychosis outcomes. Lancet Psychiatry 2: 365-367.

Light GA, Swerdlow NR, Braff DL (2007). Preattentive sensory processing is associated with cognitive and psychosocial functioning in healthy adults. J Cogn Neurosci 19: 1624-1632.

Lipton SA (2006). Paradigm shift in neuroprotection by NMDA receptor blockade: memantine and beyond. Nat Rev Drug Discov 5: $160-170$.

Ma J, Mufti A, Stan Leung L (2015). Effects of memantine on hippocampal long-term potentiation, gamma activity, and sensorimotor gating in freely moving rats. Neurobiol Aging 36: 2544-2554.

Matsuda Y, Kishi T, Iwata N (2013). Efficacy and safety of NMDA receptor antagonists augmentation therapy for schizophrenia: an updated meta-analysis of randomized placebo-controlled trials. $J$ Psychiatr Res 47: 2018-2020.

Mazinani R, Nejati S, Khodaei M (2017). Effects of memantine added to risperidone on the symptoms of schizophrenia: a randomized double-blind, placebo-controlled clinical trial. Psychiatry Res 247: 291-295.

McNally JM, McCarley RW (2016). Gamma band oscillations: a key to understanding schizophrenia symptoms and neural circuit abnormalities. Curr Opin Psychiatry 29: 202-210.

O'Donnell BF, Vohs JL, Krishnan GP, Rass O, Hetrick WP, Morzorati SL (2013). The auditory steady-state response (ASSR): 
a translational biomarker for schizophrenia. Suppl Clin Neurophysiol 62: 101-112.

Omranifard V, Rajabi F, Mohammadian-Sichani M, Maracy M (2015). The effect of add-on memantine on global function and quality of life in schizophrenia: a randomized, double-blind, controlled, clinical trial. Adv Biomed Res 4: 211.

Paraschakis A (2014). Tackling negative symptoms of schizophrenia with memantine. Case Rep Psychiatry 2014: 384783.

Perez V, Tarasenko M, Miyakoshi M, Pianka S, Makeig S, Braff $\mathrm{D}$ et al. Mismatch negativity is a sensitive and predictive biomarker of perceptual learning during auditory cognitive training in schizophrenia. Neuropsychopharmacology (in press).

Phillips KG, Cotel MC, McCarthy AP, Edgar DM, Tricklebank M, O'Neill MJ et al (2012). Differential effects of NMDA antagonists on high frequency and gamma EEG oscillations in a neurodevelopmental model of schizophrenia. Neuropharmacology 62: 1359-1370.

Pierson TM, Yuan H, Marsh ED, Fuentes-Fajardo K, Adams DR, Markello $\mathrm{T}$ et al (2014). GRIN2A mutation and early-onset epileptic encephalopathy: personalized therapy with memantine. Ann Clin Transl Neurol 1: 190-198.

Povysheva NV, Johnson JW (2016). Effects of memantine on the excitation-inhibition balance in prefrontal cortex. Neurobiol Dis 96: 75-83.

Rackelmann S, Tarasenko M, Shiluk A, Zhang W, Bismark AW, Thomas ML et al (2016). Speech-in-Noise Perception Deficits Reflect Central Auditory Dysfunction in Schizophrenia. Proceedings Society for Neuroscience: San Diego, CA, USA.

Rezaei F, Mohammad-Karimi M, Seddighi S, Modabbernia A, Ashrafi M, Salehi B et al (2013). Memantine add-on to risperidone for treatment of negative symptoms in patients with stable schizophrenia: randomized, double-blind, placebocontrolled study. J Clin Psychopharmacol 33: 336-342.

Rissling AJ, Miyakoshi M, Sugar CA, Braff DL, Makeig SM, Light GA (2014). Cortical substrates and functional correlates of auditory deviance processing deficits in schizophrenia. Neuroimage Clin 6: 424-437.

Salisbury DF, Polizzotto NR, Nestor PG, Haigh SM, Koehler J, McCarley RW (2017). Pitch and duration mismatch negativity and premorbid intellect in the first hospitalized schizophrenia spectrum. Schizophr Bull 43: 407-416.

Sheehan DV, Lecrubier Y, Sheehan KH, Amorim P, Janavs J, Weiller E et al (1998). The Mini-International Neuropsychiatric Interview (M.I.N.I.): the development and validation of a structured diagnostic psychiatric interview for DSM-IV and ICD-10. J Clin Psychiatry 59(Suppl 20): 22-33.

Sivarao DV, Chen P, Senapati A, Yang Y, Fernandes A, Benitex Y et al (2016). $40 \mathrm{~Hz}$ auditory steady-state response is a pharmacodynamic biomarker for cortical NMDA receptors. Neuropsychopharmacology 41: 2232-2240.
Sonkusare SK, Kaul CL, Ramarao P (2005). Dementia of Alzheimer's disease and other neurodegenerative disordersmemantine, a new hope. Pharmacol Res 51: 1-17.

Spencer KM, Nestor PG, Perlmutter R, Niznikiewicz MA, Klump MC, Frumin M et al (2004). Neural synchrony indexes disordered perception and cognition in schizophrenia. Proc Natl Acad Sci USA 101: 17288-17293.

Sun Y, Farzan F, Barr MS, Kirihara K, Fitzgerald PB, Light GA et al (2011). Gamma oscillations in schizophrenia: mechanisms and clinical significance. Brain Res 1413: 98-114.

Swerdlow NR (2011). Are we studying and treating schizophrenia correctly? Schizophr Res 130: 1-10.

Swerdlow NR, Bhakta S, Chou HH, Talledo JA, Balvaneda B, Light GA (2016). Memantine effects on sensorimotor gating and mismatch negativity in patients with chronic psychosis. Neuropsychopharmacology 41: 419-430.

Swerdlow NR, Light GA, Cadenhead KS, Sprock J, Hsieh MH, Braff DL (2006). Startle gating deficits in a large cohort of patients with schizophrenia: relationship to medications, symptoms, neurocognition, and level of function. Arch Gen Psychiatry 63: 1325-1335.

Swerdlow NR, van Bergeijk DP, Bergsma F, Weber E, Talledo J (2009). The effects of memantine on prepulse inhibition. Neuropsychopharmacology 34: 1854-1864.

Swerdlow NR, Tarasenko M, Bhakta SG, Talledo J, Alvarez AI, Hughes EL et al. Amphetamine enhances gains in auditory discrimination training in adult schizophrenia patients. Schizophrenia Bull (in press).

Tarasenko M, Swerdlow NR, Makeig S, Braff DL, Light GA (2014). The auditory brainstem response to complex sounds: a potential biomarker for guiding treatment of psychosis. Front Psychiatry 5: 142.

Thomas ML, Green MF, Hellemann G, Sugar CA, Tarasenko M, Calkins ME et al (2017). Modeling the cascade of deficits from early auditory information processing to psychosocial functioning in schizophrenia. JAMA Psychiatry 74: 37-46.

Thuné H, Recasens M, Uhlhaas PJ (2016). The 40-Hz auditory steady-state response in patients with schizophrenia: a metaanalysis. JAMA Psychiatry 73: 1145-1153.

Traub RD, Whittington MA, Stanford IM, Jefferys JG (1996). A mechanism for generation of long-range synchronous fast oscillations in the cortex. Nature 383: 621-624.

Veerman SRT, Schulte PFJ, Smith JD, de Haan L (2016a). Memantine augmentation in clozapine-refractory schizophrenia: a randomized double-blind, placebo-controlled crossover study. Psychol Med 46: 1909-1921.

Veerman SR, Schulte PF, Deijen JB, de Haan L (2016b). Adjunctive memantine in clozapine-treated refractory schizophrenia: an open-label 1-year extension study. Psychol Med 47: 363-375.

Zdanys K, Tampi RR (2008). A systematic review of off-label uses of memantine for psychiatric disorders. Prog Neuropsychopharmacol Biol Psychiatry 32: 1362-1374.

Supplementary Information accompanies the paper on the Neuropsychopharmacology website (http://www.nature.com/npp) 